\title{
COVID-19 Infection in Octagenarian Patients: Imaging Aspects and Clinical Correlations
}

\author{
Alessandra Marumi Emori Takahashia, b Affonso Bruno Binda do Nascimento ${ }^{a}$ \\ André Santos Barros ${ }^{\mathrm{a}}$ Márcio Barros Gaspar ${ }^{\mathrm{a}}$ Mirella Oliveira Silva ${ }^{\mathrm{a}}$ \\ Fabrício Próspero Machado ${ }^{\mathrm{a}}$ \\ aDepartment of Radiology and Diagnostic Imaging at Prevent Senior, São Paulo, Brazil; \\ ${ }^{b}$ Department of Radiology and Diagnostic Imaging at A. C. Camargo Cancer Center, São Paulo, Brazil
}

\section{Keywords}

Computed tomography · COVID-19 infection · Elderly people $\cdot$ Imaging $\cdot$ Tomographic manifestations $\cdot$ Clinical correlations

\begin{abstract}
Introduction: Computed tomography (CT) of the chest, although not a screening test or diagnosis of infection with the new coronavirus, has a fundamental role in assessing the extent of lung involvement and complications such as pleural effusion. Considering the higher morbidity and mortality of elderly patients due to this infection, the objective of this study was to evaluate the imaging aspects and clinical correlations of an extreme age ( $\geq 80$ years) with a confirmed diagnosis for COVID-19. Methods: This was a retrospective and single-center cohort study. CT scans were categorized qualitatively and quantitatively. In the first case, 3 descriptors were used to describe CT findings: "compatible" (findings of greater specificity for COVID-19: opacities with attenuation in ground glass with peripheral and bilateral distribution, with rounded morphology, with or without
\end{abstract}

consolidations, crazy-pavement aspect, inverted halo sign, or organizing pneumonia findings), "doubtful" (findings not specific or unusual for COVID-19: opacities with attenuation in ground glass with nonrounded morphology, central, diffuse, or unilateral distribution, with or without consolidation, lobar or segmental consolidation without ground-glass opacity, small centrilobular nodules with the appearance of "tree-in-bud," excavations, pleural effusion, and thickening of interlobular septa), and "negative" (absence of pneumonia signs). For the quantitative assessment, which referred to the extent of pulmonary involvement, a tomographic severity classification was used: grade 1 (lung involvement $\leq 25 \%$ ), grade 2 (pulmonary involvement between 26 and $50 \%$ ), and grade 3 (pulmonary involvement $>50 \%$ ). Results: A total of 138 patients were evaluated, with an average age of 86.2 years ( 84 women and 34 men). The mean time interval between onset of symptoms and tomography was 5.63 days. The most prevalent comorbidity was systemic arterial hypertension (81.2\%). Compatible, doubtful, and negative tests were 117 (84.7\%), 20 (14.4\%), and 1 (0.7\%), respectively. As for compatible exams, the most common findings were opacities in peripheral ground glass and rounded mor-
Correspondence to:

Alessandra Marumi Emori Takahashi, alessandra.emori@gmail.com 
phology, followed by crazy paving. The prevalence of pleural effusion was $28.2 \%$ and consolidation was $63.7 \%$, and none of these findings were influenced by the duration of symptoms ( $p=0.08$ and $p=0.2$, respectively). The exams classified as grade 1, grade 2 , and grade 3 were $57(41.6 \%)$, $46(33.6 \%)$, and $34(24.8 \%)$, respectively. There were statistically significant associations between the classification of tomographic severity and outcomes such as invasive ventilation ( $p=0.004)$, admission to the intensive care unit ( $p<$ $0.001)$, and death $(p<0.001)$. Discussion/Conclusion: Our results show that patients $\geq 80$ years old present tomographic manifestations similar to those described for the general population (ground-glass opacities and "crazy paving") and that the extent of lung involvement is associated with the need for intensive care, invasive ventilation, and death. Although the literature describes an association between the stage of the disease and the appearance of consolidations and pleural effusion, this correlation was not observed in our study, which may suggest that this age-group is more predisposed to the appearance of such findings, typically described in the more advanced stages of infection.

(c) 2021 S. Karger AG, Basel

\section{Introduction and Objectives}

The pandemic caused by the new coronavirus, whose epicenter was in the city of Wuhan, China, in December 2019, generated a progressive demand in health services due to its high transmissibility [1]. Although its lethality is low (up to $3.5 \%$ ), it is estimated that up to one-fifth of those infected develop severe pneumonia and up to $10 \%$ require intensive care. It is known that the elderly population is most susceptible to morbidity and mortality [2], especially when there is an association with comorbidities, which are common in this age-group. This explains, in part, the increased need for social isolation in this group by health authorities.

Computed tomography (CT) of the chest is not routinely recommended for screening or initial diagnosis of COVID-19 [3] and sensitivity and specificity are quite variable in the literature, with values from 60 to $98 \%$ and $25-53 \%$, respectively [4], most likely due to the retrospective nature of most studies. Barbosa et al. [5], in a cohort involving 91 exams, found different diagnostic accuracy values depending on tomographic findings. When presented with a typical exam for COVID-19, the accuracy was $79.1 \%$, while if a typical or indeterminate exam for COVID-19 was present, the rate was $70.3 \%$. In addition, tomography is of great value for estimating the extent of pulmonary involvement, which has been shown to be associated with clinical severity, as well as for detecting complications such as pleural effusion.

The World Health Organization defines elderly as individuals aged 60 years and over and recognizes that, despite this minimum threshold, it is important to consider that chronological age is not an accurate marker for the changes that accompany the aging process [6]. The frail state of an elderly person is a prolonged process of disability, vulnerability, and predisposition to functional decline. The older the age, the more prevalent is the frail state of an elderly person, the greater the chance of comorbidities, the lower the cardiopulmonary reserve, and consequently, the greater the chance of unfavorable outcomes in the face of infection with the new coronavirus.

Considering the particularities of an extreme agegroup, the aim of this study was to evaluate the tomographic manifestations of patients aged 80 years or older with a confirmed diagnosis for infection with the new coronavirus, seeking to describe the most common manifestations and possible atypical findings in these patients. In addition, we sought to assess the correlation between imaging findings with outcomes such as invasive ventilation and death, as well as the association of the duration of symptoms in the presence of pleural effusion and consolidations, commonly found in the more advanced stages of infection.

\section{Materials and Methods}

This was a cross-sectional, retrospective, observational, singlecenter study, started after approval by the Ethics Committee under code 4.071.410. As this was a retrospective study, free and informed consent was waived. Data were entered using Microsoft Excel Software through the review of documents from electronic medical records and the Picture Archiving and Communication Systems reporting system.

Patients aged 80 years or older who underwent chest CT at the time of initial medical care for flu-like symptoms and who had a confirmed diagnosis of COVID-19 through reverse transcription polymerase chain reaction(RT-PCR) were selected. Material for RT-PCR was obtained by collecting a nasopharynx swab on the same day as the CT scan. The period covered in this study was March and April 2020.

Multidetector tomographs with a low radiation dose protocol were used and the images were acquired during deep inspiration, without the administration of intravenous contrast. The cutting thickness used was $0.625-1.25 \mathrm{~mm}$, with the same increment.

The images were analyzed by at least 2 expert radiologists (blinded by clinical and laboratory data) according to the categorization recommended by the American College of Radiology and the Radiology Society of North America, which proposes 4 radiological standards [7]: 
Fig. 1. Images in an 89-year-old man, with systemic arterial hypertension and obesity. Time interval between onset of symptoms and tomography was 7 days. Unenhanced chest CT scan with typical features for COVID-19 infection. a-c Axial section showing periferal and multifocal ground-glass opacity (arrows). d-f Coronal section showing pulmonary extension under $25 \%$ (Grade 1). CT, computed tomography.
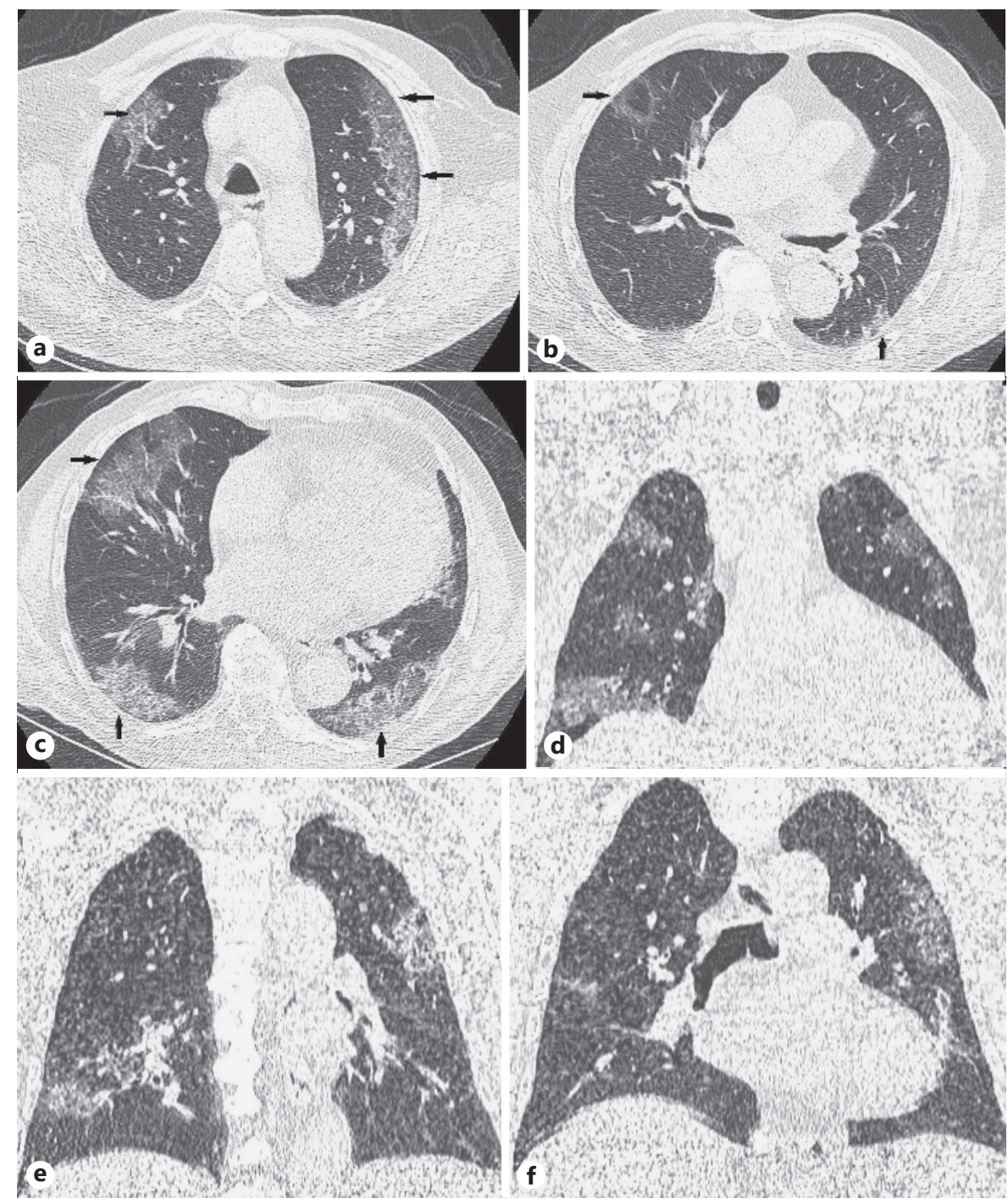

- Typical: findings of greater specificity for COVID-19. Opacities with attenuation in ground glass with peripheral and bilateral distribution, with rounded morphology, with or without consolidations, crazy-pavement aspect, inverted halo sign or organizing pneumonia findings.

- Undetermined: findings not specific to COVID-19. Absence of typical findings and presence of opacities with attenuation in ground glass with nonrounded morphology, central, diffuse, or unilateral distribution, with or without consolidation.

- Atypical: unusual or unreported findings in COVID-19, making it necessary to consider differential diagnoses. Absence of typical or indeterminate findings and presence of lobar or segmental consolidation without ground-glass opacity, small centrilobular nodules with the appearance of "tree-in-bud," excavations, pleural effusion, and thickening of interlobular septa.

- Negative: absence of pneumonia signs.

In our study, findings of greater specificity for COVID-19 (typical standard) were called "compatible tomography for COVID-19." For simplification, we grouped findings not specific (un-

determined standard) or unusual/unreported findings (atypical standard) in what are called "doubtful tomography for COVID-19." We called "negative tomography for COVID-19" exams with no findings of pneumonia signs.

In addition to the descriptive classification, we also used a tomographic severity classification, which is based on stratification by the extent of pulmonary impairment proposed by the British Society of Thoracic Imaging (British Society of Thoracic Imaging), which is divided into $4^{\circ}[8]: \leq 25,26-50,51-75$, and $>75 \%$. In our study, this classification was adapted, so that the last 2 stratifications were included in only 1 group, as we considered that the impairment of more than half of the lung parenchyma associated with advanced age would be an indication of greater severity. Thus, the classification of tomographic severity was composed of 3 categories:

- Grade 1: lung involvement $\leq 25 \%$;

- Grade 2: pulmonary involvement between 26 and 50\%;

- Grade 3: pulmonary involvement $\geq 50 \%$.

It is worth mentioning that this classification was applied to exams categorized as compatible or doubtful for COVID-19. 
Fig. 2. Images in an 88-year-old woman, with systemic arterial hypertension and obesity. Time interval between onset of symptoms and tomography was 10 days. Unenhanced chest CT scan with typical features for COVID-19 infection. a-c Axial section showing diffuse ground-glass opacities (arrows), and visible intralobular lines. d-f Coronal section showing pulmonary extension between 26 and 50\% (Grade 2). CT, computed tomography.

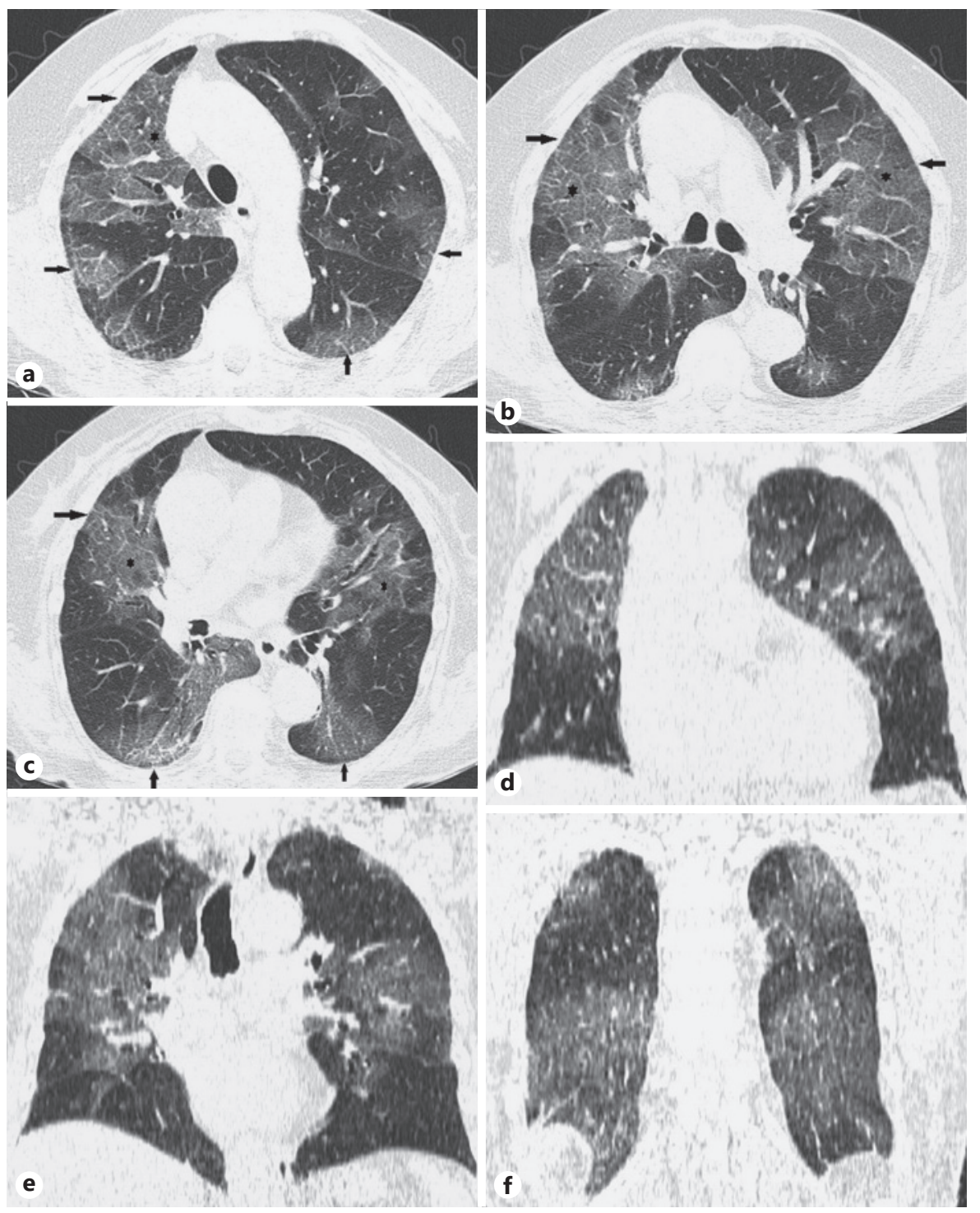

Descriptive analysis of epidemiological and clinical data was performed. Information regarding the type of treatment adopted was also collected and grouped as follows: group A, home treatment; group $B$, treatment in an inpatient unit, and group $C$, treatment in an intensive care unit. Within the latter categorization, patients were further divided according to the need for invasive ventilation. Tomographic findings were correlated with clinical variables such as treatment modality, need for invasive ventilation, and death.

Patients whose tomographic images or electronic medical record data were not available were excluded from this study. The information obtained was stored in a database for statistical analysis using the software R version 3.6.0 (copyright (C) 2019; The R Foundation for Statistical Computing). Descriptive analyses of variables were performed, with distributions of absolute $(n)$ and relative (\%) frequencies for qualitative variables, and the main summary measures, such as mean, standard deviation, median, minimum and maximum values, for quantitative variables. For the study of qualitative variables, $2 \times 2$ tables were used to assess statistical significance using a Pearson's $\chi^{2}$ test with Yates correction or Fisher's exact test, when indicated. For quantitative variable evaluation in relation to the classification of tomography, a Student's $t$ test was used. In order to assess the influence of time on the appearance of certain tomographic findings, logistic regression was performed, in which the odds ratio value was evaluated, Finally, to assess the agreement between the 2 observers in the tomographic severity classification, the kappa coefficient was used. Results with a type 1 error probability $\leq 5 \%$ ( $p \leq$ $0.05)$ were considered statistically significant.

\section{Results}

A total 138 patients were evaluated, most of them female, and no patient was excluded from this study. Age ranged between 81 and 100 years and the longest interval 
Fig. 3. Images in an 85-year-old woman, with systemic arterial hypertension. Time interval between onset of symptoms and tomography was 6 days. Unenhanced chest CT scan with typical features for COVID-19 infection. a-c Axial section showing bilateral, multifocal rounded (asterisks), and peripheral ground-glass opacity (arrows). d-f Coronal section showing pulmonary extension $>50 \%$ (Grade 3 ). CT, computed tomography.
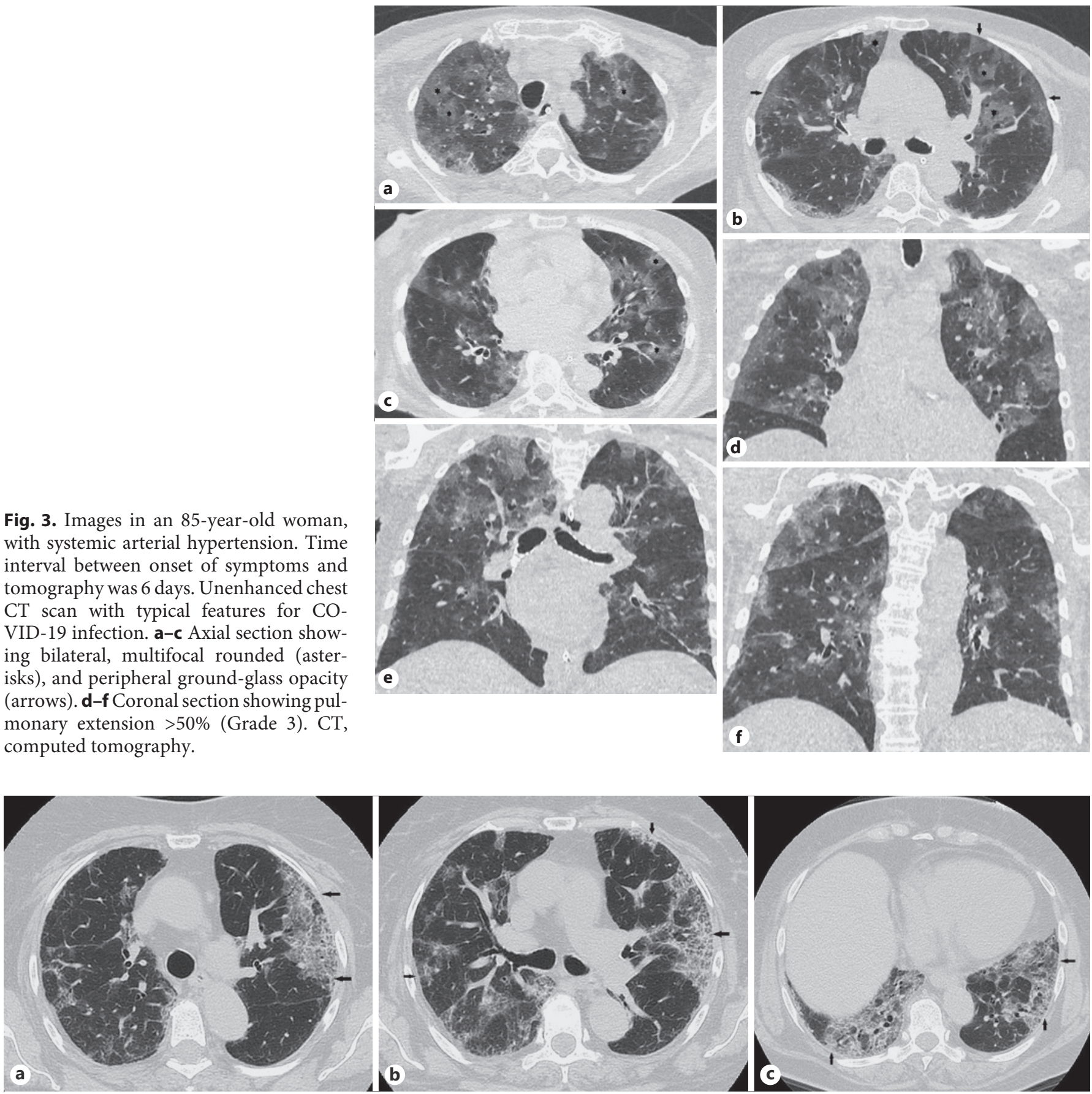

Fig. 4. Images in an 83-year-old woman, with systemic arterial hypertension and obesity. Time interval between onset of symptoms and tomography was 19 days. Unenhanced chest CT scan with typical features for COVID-19 infection. a-c Axial section show-

ing bilateral and multifocal ground-glass opacity (arrows) with superimposed interlobular septal thickening and visible intralobular lines ("crazy paving"). CT, computed tomography.

between the onset of symptoms and the performance of chest CT was 30 days. Regarding comorbidities, the most prevalent was systemic arterial hypertension and the least common was obesity.

Regarding CT scans, we had the following numbers of compatible, doubtful, and negative tests, respectively: $84.7 \%$ (117/138), $14.4 \%$ (20/138), and 0.7\% (1/138). As for tomographic severity (due to the extent of lung in- 
Table 1. Epidemiological and clinical characteristics of patients

\begin{tabular}{|c|c|c|c|c|}
\hline & $\begin{array}{l}\text { Total } \\
(N=138)\end{array}$ & $\begin{array}{l}\text { Doubtful } \\
(N=20)\end{array}$ & $\begin{array}{l}\text { Compatible } \\
(N=117)\end{array}$ & $p$ value ${ }^{*}$ \\
\hline \multicolumn{5}{|l|}{ Age, year } \\
\hline Median (min. and max.) & $85(81 ; 100)$ & $84.5(81 ; 94)$ & $85(81 ; 100)$ & \multirow{2}{*}{0.69} \\
\hline Mean \pm standard deviation & $86.2 \pm 4.22$ & $85.8 \pm 4.3$ & $85 \pm 4.3$ & \\
\hline \multicolumn{5}{|l|}{ Gender, $n(\%)$} \\
\hline Female & $84(60.9)$ & $12(60)$ & $71(60.7)$ & \multirow{2}{*}{1.0} \\
\hline Male & $54(39.1)$ & $8(40)$ & $46(39.3)$ & \\
\hline \multicolumn{5}{|l|}{ Time interval, days } \\
\hline Median (min. and max.) & $4(0 ; 30)$ & $1(0 ; 28)$ & $5(0 ; 32)$ & \multirow{2}{*}{0.79} \\
\hline Mean \pm standard deviation & $5.63 \pm 5.52$ & $5.2 \pm 7.89$ & $5.7 \pm 5.07$ & \\
\hline \multicolumn{5}{|l|}{ Comorbidities, $n(\%)$} \\
\hline Systemic arterial hypertension & $112(81.2)$ & $14(70)$ & $98(83.8)$ & 0.25 \\
\hline Diabetes mellitus & $49(35.5)$ & $8(40)$ & $41(35)$ & 0.86 \\
\hline Cardiovascular disease & $49(35.5)$ & $8(40)$ & $41(35)$ & 0.86 \\
\hline Cerebrovascular disease & $34(24.6)$ & $7(35)$ & $27(23.1)$ & 0.27 \\
\hline Smoking & $25(18.1)$ & $5(25)$ & $20(17.1)$ & 0.36 \\
\hline Asthma or COPD & $21(15.2)$ & $3(15)$ & $20(17)$ & 1.0 \\
\hline Body mass index $>30 \mathrm{~kg} / \mathrm{m}^{2}$ & $20(14.5)$ & $3(15)$ & $17(14.5)$ & 1.0 \\
\hline \multicolumn{5}{|l|}{ Treatment type, $n(\%)$} \\
\hline Home & $19(13.7)$ & $2(10)$ & $17(14.5)$ & \multirow{3}{*}{$0.73^{* *}$} \\
\hline Ward & $84(60.8)$ & $14(70)$ & $69(59)$ & \\
\hline ICU & $35(25.3)$ & $4(20)$ & $31(26.5)$ & \\
\hline
\end{tabular}

ICU, intensive care unit. ${ }^{*} \chi^{2}$ test. ${ }^{* *}$ Student $t$ test.

Table 2. Agreement between the 2 evaluators (kappa $=0.82$; agreement $=88.3 \%, n=137)$ in the classification of tomographic severity

\begin{tabular}{llcl}
\hline Evaluator 1 & \multicolumn{3}{l}{ Evaluator 2 } \\
& Grade 1, $n(\%)$ & Grade 2, $n(\%)$ & Grade 3, $n(\%)$ \\
\hline Grade 1 & $53(38.7)$ & $3(2.2)$ & $0(0.0)$ \\
Grade 2 & $4(2.9)$ & $42(30.7)$ & $1(0.7)$ \\
Grade 3 & $1(0.7)$ & $7(5.1)$ & $26(19.0)$ \\
\hline
\end{tabular}

volvement), the tests classified as grade 1 , grade 2 , and grade 3 were $57(41.6 \%), 46$ (33.6\%), and $34(24.8 \%)$, respectively (Fig. 1-3).

There was no statistically significant difference in terms of epidemiological and clinical characteristics in the groups of patients with tests classified as positive and doubtful, as shown in Table 1. For statistical purposes, the only patient with a negative test was disregarded from these calculations. The agreement between the 2 evaluators regarding tomographic severity classification was almost perfect, as shown by the kappa coefficient in Table 2 .
Of the 117 exams classified as compatible, 111 (94.8\%) were presented with ground-glass opacities, either with rounded morphology and multifocal distribution, or with nonrounded morphology with peripheral and bilateral distribution, with an association with "crazy paving" in 83 (70.9\%) exams (Fig. 1-4). Absence of ground-glass opacities was observed in only 6 patients $(5.1 \%)$, who were classified as compatible due to the presence of an inverted halo sign or signs of organizing pneumonia (Fig. 5). Consolidations and pleural effusion were present in $63.2 \%(74 / 117)$ and $23.9 \%$ (11/28), respectively (Fig. 6). Table 3 shows all these findings.

Although 75\% (15/20) of the tomographies classified as doubtful presented ground-glass opacities, they were of central or hilar distribution, of nonrounded morphology. Approximately $1 / 3$ of the patients $(7 / 20)$ presented consolidations with air bronchograms, findings suggestive of pneumonia of non-viral etiology (Fig. 7). Of these, there was an overlap with Staphylococcus bacterial infection in 1 patient, confirmed by blood culture; in the remaining 6 patients' blood samples were either not collected or they did not grow bacteria. About 55\% (11/20) had pleural effusion, most of them bilateral. Pulmonary excavation was characterized in 1 patient, who also had a 

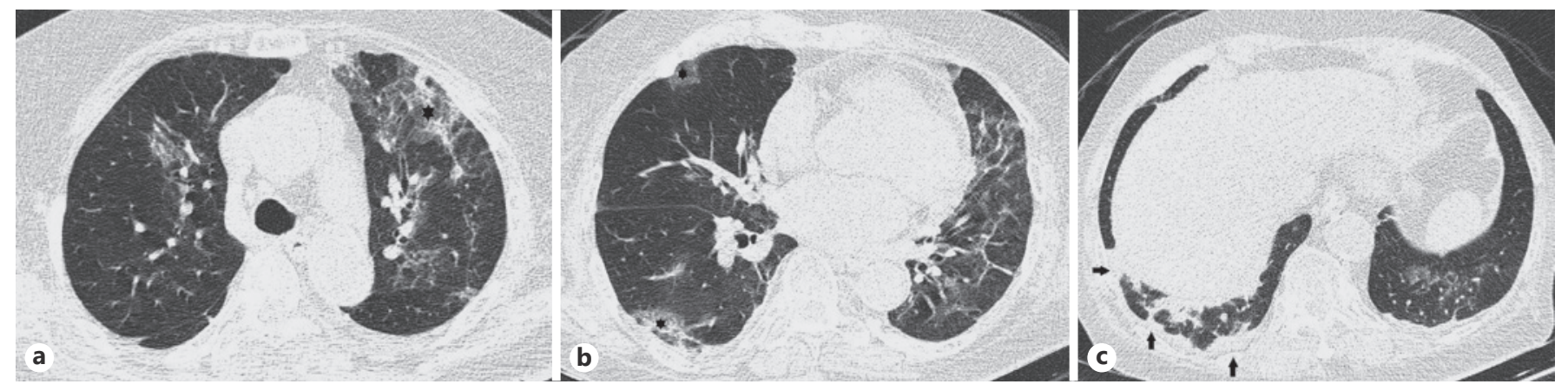

Fig. 5. Images in an 84-year-old woman with systemic arterial hypertension and diabetes. Time interval between onset of symptoms and tomography was 2 days. Unenhanced chest CT scan shows

organizing pneumonia features. a-c Posterior and peripheral ground-glass opacities (asterisks) with consolidations areas (arrows). CT, computed tomography.

Fig. 6. Images in an 83-year-old smoker man with systemic arterial hypertension. Time interval between onset of symptoms and tomography was only 1 day. Unenhanced chest CT scan show typical COVID-19 infection sumperimposed with bacterial pneumonia. a, b Axial section showing central and peripheral groundglass opacities (asterisks), segmental consolidation (arrowhead), and pleural effusion. Air bronhograms are observed in the right lower lobe, suggestive of nonviral etiology, which was confirmed by blood culture. CT, computed tomography.

Table 3. Tomographic characteristics of positive and doubtful exams
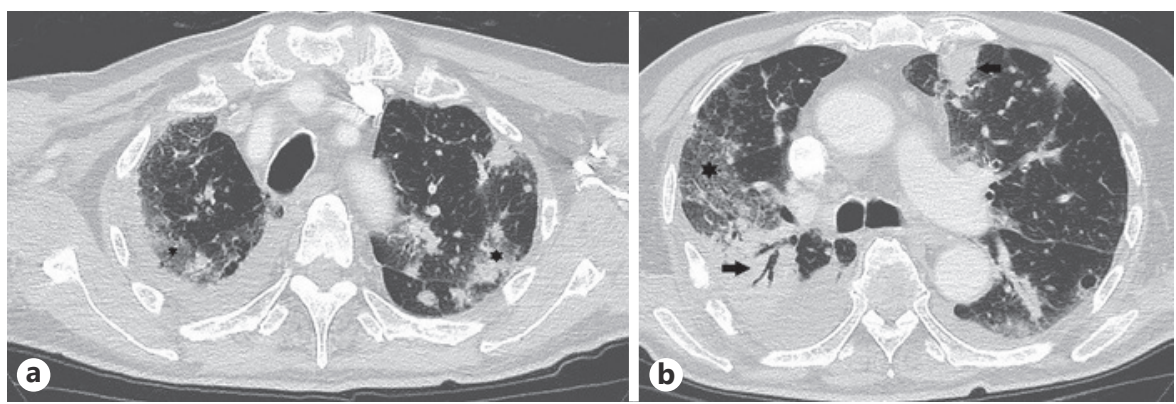

Doubtful-14.4\% (20/138)

Descriptive tomographic findings, \% $(n / N)$

Nonrounded/nonperipheral ground-glass

Consolidating opacities

Centrilobular nodules ("tree-in-bud")

$70(14 / 20)$

$5(1 / 20)$

Excavation

$5(1 / 20)$

Pleural effusion

Tomographic severity (pulmonary extension), $n$ (\%)

Grade 1

Grade 2

Grade 3

$55(11 / 20)$

$20(100)$

0

0

Compatible-84.7\% (117/138)

Descriptive tomographic findings, $\%(n / N)$

Rounded, peripheral, bilateral ground-glass

$94.8(111 / 117)$

Crazy paving

Inverted halo sign

$70.9(83 / 117)$

$0.8(1 / 117)$

$4.2(5 / 117)$

Signs of organizing pneumonia

$63.2(74 / 117)$

Pleural effusion

$23.9(28 / 117)$

Tomographic severity (pulmonary extension), $n$ (\%)

Grade 1

$37(30.8)$

Grade 2

$46(40.2)$

Grade 3

$34(29)$
COVID-19 Infection in Octagenarian Patients
Gerontology 2022;68:261-271

DOI: $10.1159 / 000516166$ 
Fig. 7. Images in an 84-year-old smoker woman. Time interval between onset of symptoms and tomography was 7 days. Unenhanced chest CT scan with doubtful features for COVID-19 infection. a, b Axial section showing hilar/central groundglass opacity (arrows), consolidation with air bronchograms in the right lower lobe (asterisks) and pleural effusion. CT, computed tomography.

Fig. 8. Images in an 81-year-old smoker woman. Time interval between onset of symptoms and tomography was 10 days. Unenhanced chest CT scan with doubtful features for COVID-19 infection. a, b Axial section, pulmonary window showing cavitation in left superior lobe (white asterisk), pulmonary mass in right lower lobe (arrows). c, d Axial section, soft tissue window showing mediastinal lymph node enlargement (black asterisks). All those findings are suggestive of neoplasm etiology. CT, computed tomography.

Fig. 9. Images in an 87-year-old woman. Time interval between onset of symptoms and tomography was 3 days. Unenhanced chest CT scan with negative features for COVID-19 infection. a Axial section, soft tissue window showing right loculated pleural effusion associated with nodular thickening of mediastinal pleura. b Axial section, pulmonary window showing no significant alteration in pulmonary parenchyma. CT, computed tomography.
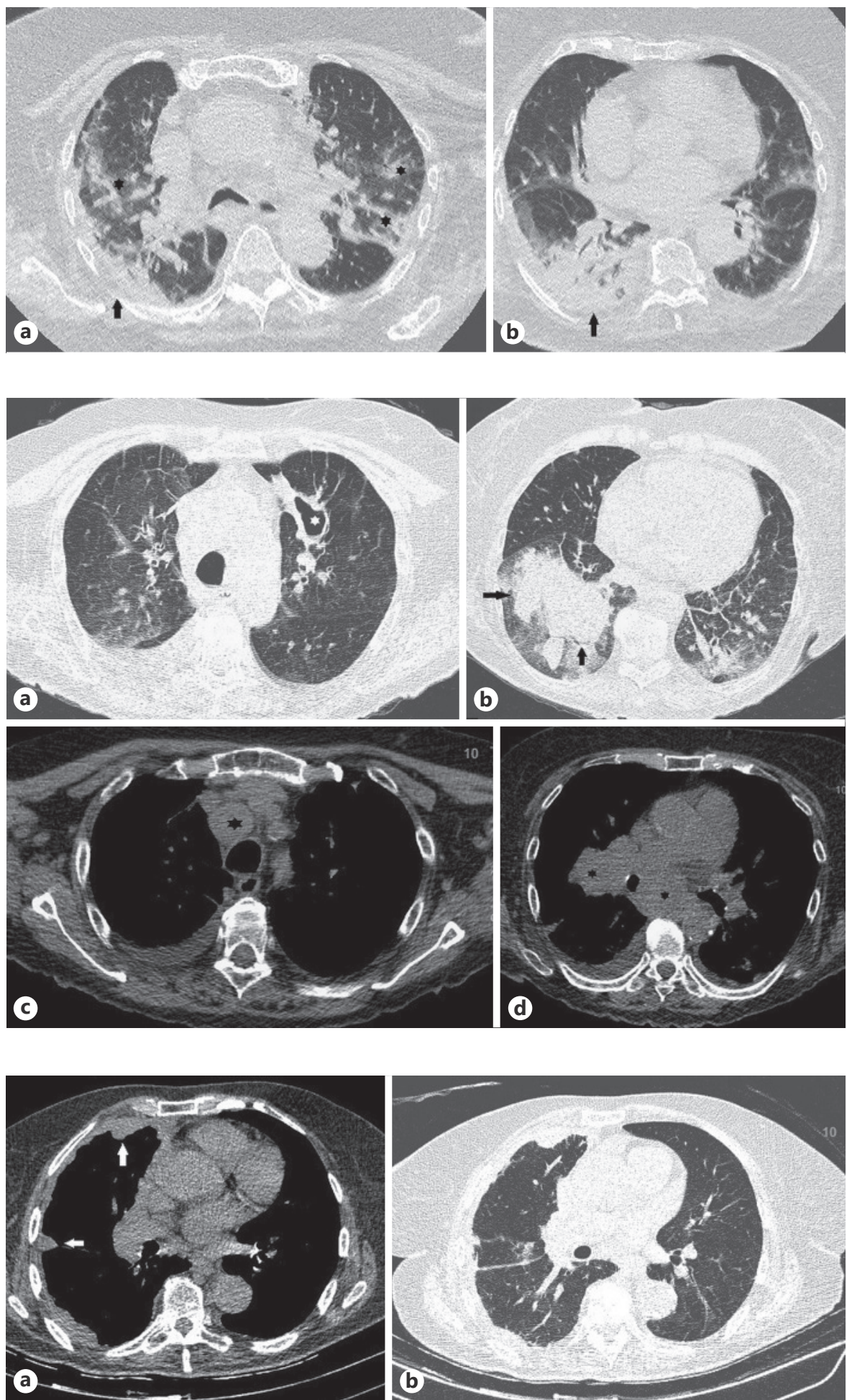

pulmonary mass in the right lower lobe and mediastinal lymph node enlargement, findings suggestive of neoplasia (Fig. 8). Only 1 patient presented findings suggestive of a small airway infectious process, represented by small branched centrilobular nodular opacities, with the appearance of a "tree-in-bud."

Regarding the patient with a negative finding for $\mathrm{CO}$ VID-19, the examination showed only unilateral loculated 
Table 4. Association between tomographic severity and invasive ventilation and death

\begin{tabular}{|c|c|c|c|}
\hline $\begin{array}{l}\text { Tomographic } \\
\text { severity }\end{array}$ & $\begin{array}{l}\text { Total } \\
(N=137), n(\%)\end{array}$ & $\begin{array}{l}\text { MV } \\
\text { No, } n(\%) / Y e s, n(\%)\end{array}$ & $\begin{array}{l}\text { Death } \\
\text { No, } n(\%) / \text { Yes, } n(\%)\end{array}$ \\
\hline Grade 1 & $57(41.6)$ & $46(50.5) / 11(23.9)$ & $33(61.1) / 24(28.9)$ \\
\hline Grade 2 & $46(33.6)$ & $29(31.9) / 17(37)$ & $16(29.6) / 30(36.1)$ \\
\hline Grade 3 & $34(24.8)$ & $16(17.6) / 18(39.1)$ & $5(9.26) / 29(34.9)$ \\
\hline$p$ value* & & 0.004 & $<0.001$ \\
\hline
\end{tabular}

MV, mechanical ventilation. ${ }^{*} \chi^{2}$ test. pleural effusion, associated with foci of nodular thickening of the mediastinal pleura, findings possibly related to neoplasia, as illustrated in Figure 9. Table 4 summarizes the descriptive tomographic findings according to the classification and the extent of pulmonary involvement.

When performing an association between the descriptive classification of the tomographic examination and the need for mechanical ventilation (MV), we did not find any statistically significant difference between groups $(p=0.5)$. However, when we performed a correlation between tomographic severity with the need for MV and death, we noticed that there was a statistically significant difference, indicating that the greater the degree of pulmonary involvement, the greater the association with MV and death (Table 4). In addition, there was a statistically significant correlation between the degree of pulmonary involvement and the adopted treatment type, with $p<$ 0.001 . Regarding the presence of consolidations and pleural effusion, findings most commonly described in the advanced stages of infection (after 1 week of symptoms), we observed that there was no influence of time between symptoms and findings relating these 2 variables, as shown in Table 5 below:

\section{Discusion and Conclusion}

The importance of this work centers on the evaluation of CT scans of a population that belongs to an extremely vulnerable age-group with confirmed infection for COVID-19. Our results showed that, of the 138 patients with positive RT-PCR for COVID-19, 117 (85\%) presented compatible tomography for this infection, which demonstrates that the descriptors suggested by the American College of Radiology and Radiology Society of North America showed a good correlation when applied to patients with an age greater than or equal to 80 years. The most common findings in this examination category were ground-glass opacities with peripheral distribution and
Table 5. Logistic regression to assess the influence of the time interval on variables such as consolidation and pleural effusion

\begin{tabular}{llll}
\hline Variable & $p$ value & OR & CI 95\% (OR) \\
\hline Consolidation & 0.2 & 1.04 & $0.98-1(13)$ \\
Pleural effusions & 0.08 & 1.06 & $0.99-1(13)$ \\
\hline
\end{tabular}

OR, odds ratio.

rounded morphology (80.4\%), followed by "crazy paving" (60.1\%). Chen et al. [9], also found both findings the most common in their sample, which included patients between 4 and 88 years old with confirmed infection by COVID-19 and, when comparing different age-groups, realized that such descriptors were more common in patients aged 45 years or older, with $p<0.001$. Chung et al. [10], when retrospectively evaluating 21 patients, found a similar value ( $85 \%)$ to our study regarding ground-glass opacities; however, "crazy paving" was characterized in only $19 \%$ of the exams. In a study by Zhou et al. [11], involving patients between 20 and 91 years old, groundglass opacities (with or without consolidations) and "crazy paving" were the most prevalent findings both in the initial stage of the disease (first 3 days of symptoms) and in the most advanced stage (4-7 days of symptoms), with a rate of $97 \%(33 / 34)$ and $23.5 \%(8 / 34)$ for the first group and $96 \%(27 / 28)$ and $28 \%(8 / 28)$ for the second group, respectively. In addition, it found the halo sign in almost $21 \%$ of early stage examinations (7/34) and, although this study has signaled the importance of this sign as an initial finding of coronavirus infection, we have not found other studies with similar results in the literature. In our series, no patient had the halo sign.

Consolidating aspect opacities are postulated to be more common in older patients [1] and in the more advanced stages of infection [12], after about 9 days of symptom onset [13]. In fact, about $63 \%(88 / 138)$ of our patients presented consolidations, most of them (94.3\%) 
in coexistence with ground-glass opacities (either centrally or peripherally). In the literature, we found variable consolidation rates, such as 2.9 [11] and 28\% [10], with the average age of these studies being 44 and 51 years, respectively. In our sample, the mean age was 85 years. In addition, we observed that there was no association between the duration of symptoms and the presence of consolidations ( $p=0.2$ ), which may suggest that this finding was common in our sample due to the advanced age of the patients. However, these data are insufficient to state that advanced age was a risk factor for consolidations.

Similar to consolidations, pleural effusion was also associated with more advanced stages of infection; however, there was also no influence of time of symptoms on the appearance of this complication $(p=0.08)$. Although Salehi et al.'s [14] systematic review cited pleural effusion as an unusual finding in the setting of coronavirus infection, in our sample this finding was relatively common, present in 39 patients $(28 \%)$, a rate significantly higher than studies performed by Chung et al. [10] and Bernheim et al. [13], with rates of $0 \%(0 / 21)$ and $0.8 \%(1 / 121)$, respectively.

Only 1 patient in our study $(0.7 \%)$ had a negative CT scan for COVID-19, belonging to an 87-year-old patient who underwent the examination on the third day of symptoms. In the study by Chung et al. [10], of the 21 symptomatic patients evaluated, 3 (14\%) had a normal exam; however, we do not have information on the duration of symptoms of these patients. Bernheim et al. [13], when evaluating 121 exams of symptomatic patients, found that $22 \%$ (27 patients) were normal exams, suggesting that the more advanced the stage of the disease, the lower the rate of unchanged exams. For example, of the 36 exams of patients with an onset of symptoms up to 2 days, the rate of negative exams was $56 \%(20 / 36)$. The maximum age of the patients involved in the 2 studies cited above was 77 and 80 years, respectively, less than the average age of our study (86 years), which could support the hypothesis that the older the age, the lower the chance of negative exams.

In our series, we did not observe an association between the exam category (compatible or doubtful) and the need for MV. In the literature, there are no studies that have made such a comparison. On the other hand, regarding the extent of pulmonary involvement, we observed a statistically significant association with clinical severity: the higher the degree, the greater the association with admission to the ICU, the need for MV, and death, with $p<0.001, p=0.004$ and $p<0.001$, respectively.

This study has some limitations. First, it was restricted to a qualitative description of image aspects in octagenar- ian patients with COVID-19 infection. There are several articles with similar analysis in literature. Our data do not allow for a robust quantitative description. We believe that future studies with a larger sample size would be necessary and more valuable. However, the strength of this study is that there has been no previous report of a cohort involving specifically this extreme of age ( $>$ ou $=80$ years). Another relevant point of this article was to show association between extent of lung involvement and clinical severity (need for intensive care, invasive ventilation, and death) specifically in this group of patients. Another limitation is that patients with very advanced age have difficulty performing the exam due to positioning or difficulty in performing deep inspiration, especially in the presence of a pulmonary condition, which presents difficulty for the assessing radiologist due to movement artifacts during the acquisition of the images. In addition, the presence of fibrosis or pulmonary emphysema, not uncommon at this age, also reduces the sensitivity of the test. It is worth noting that all patients categorized as doubtful tomography for COVID-19 received a grade 1 regarding tomographic severity, which may indicate a certain limitation in applying this classification to doubtful exams.

Our results show that, in general, patients aged 80 years or older have tomographic manifestations similar to those described for the general population (groundglass opacities and "crazy paving") and that the extent of lung involvement (tomographic severity) is associated with the need for intensive care, invasive ventilation, and death. Although the literature describes an association between the stage of the disease and the appearance of consolidations and pleural effusion, this correlation was not observed in our study, which may suggest that this age-group is more predisposed to the appearance of such findings, typically described in more advanced stages of infection. Future studies, involving a larger number of patients and with chronological tomography analysis according to the stage of the disease, may provide more information for a better understanding of pulmonary manifestations in this vulnerable population in coronavirus pandemic.

\section{Acknowledgments}

The authors thank Tatiana Figueiredo Guimarães Ribeiro, Ricardo Arroyo Rstom, Edivaldo Nery de Oliveira Filho, Talita Rombaldi Pereira, Silvio Fontana Velludo, Paula Nicole Vieira Pinto Barbosa, and Maria Fernanda Arruda Almeida for the contribution in this article. We also thank Juliana Luz Passos Argenton for the contribution at the statistical analyses. 


\section{Statement of Ethics}

Institute Review Board approval was obtained from Dante Pazzanese of Cardiology Institute Ethical Committee; CAAE: 32408920.2.0000.5462. This article is a retrospective and observational study and hence written informed was waived by the Institute Review Board.

\section{Conflict of Interest Statement}

The authors have no conflicts of interest to declare.

\section{Funding Sources}

The authors state that this study received no funding.

\section{Author Contributions}

All the authors have met the ICMJE authorship criteria and contributed to the conception of this work. Alessandra Marumi Emori Takahashi contributed to the study design, data collection, data analysis, results interpretation, discussion on results, and wrote the manuscript. Affonso Bruno Binda do Nascimento contributed to the study design, data collection, and discussion on results. André Santos Barros contributed to the statistical methods, data analyses, and data collection. Márcio Barros Gaspar contributed to the statistical methods, data analyses, and data collection. Mirella Oliveira Silva contributed to the data analyses and data collection. Fabrício Próspero Machado conceived, designed, and supervised the project. All the authors read and approved the final manuscript.

\section{References}

1 Jin Y-H, Cai L, Cheng Z-S, Cheng H, Deng T, Fan YP, et al. A rapid advice guideline for the diagnosis and treatment of 2019 novel coronavirus (2019-nCoV) infected pneumonia (standard version). Mil Med Res. 2020 Feb 6; $7(1): 4$.

2 Liu K, Chen Y, Lin R, Han K. Clinical features of COVID-19 in elderly patients: a comparison with young and middle-aged patients. J Infect. 2020;80(6):e14-8.

3 Kong W, Agarwal PP. Chest imaging appearance of COVID-19 infection. Radiol Cardiothorac Imaging. 2020 Feb 13;2(1):e200028.

4 Fang Y, Zhang H, Xie J, Lin M, Ying L, Pang $\mathrm{P}$, et al. Sensitivity of chest CT for COVID-19: comparison to RT-PCR. Radiology. 2020 Feb 19:200432.

5 Barbosa PNVP, Bitencourt AGV, Miranda GD, Almeida MFA, Chojniak R. Acurácia da TC de tórax no diagnóstico de infecção por SARS-CoV-2: experiência inicial em um centro oncológico. Radiol Bras. 2020.
6 Heikkinen RL; OMS. Growing older. Staying well. Ageing and physical activity in everyday life. Genebra: Organização Mundial da Saúde; 1998.

7 Simpson S, Kay FU, Abbara S, Bhalla S, Chung $\mathrm{JH}$, Chung M, et al. Radiological Society of North America expert consensus document on reporting chest $\mathrm{CT}$ findings related to $\mathrm{CO}$ VID-19. Endorsed by the Society of Thoracic Radiology, the American College of Radiology, and RSNA. Radiol Cardiothorac Imaging. 2020;2(2):e200152.

8 British Society of Thoracic Imaging. Thoracic imaging in COVID-19 infection: guidance for the reporting radiologist, version 2. 2020 Mar. Available from: https://www.bsti.org.uk/media/resources/files/BSTI_COVID19_Radiology_Guidance_version_2_16.03.20.pdf.

9 Chen Z, Fan H, Cai J, Li Y, Wu B, Hou Y, et al. High-resolution computed tomography manifestations of COVID-19 infections in patients of different ages. Eur J Radiol. 2020; 126:108972.
10 Chung M, Bernheim A, Mei X, Zhang N, Huang $M$, Zeng $X$, et al. CT imaging features of 2019 novel coronavirus (2019-nCoV). Radiology. 2020 Apr;295(1):202-7.

11 Zhou Z, Guo D, Li C, Fang Z, Chen L, Yang $\mathrm{R}$, et al. Coronavirus disease 2019: initial chest CT findings. Eur Radiol. 2020;30(8):4398406.

12 Pan F, Ye T, Sun P, Gui S, Liang B, Li L, et al. Time course of lung changes on chest CT during recovery from 2019 novel coronavirus (COVID-19) pneumonia. Radiology. 2020 Feb 13:200370.

13 Bernheim A, Mei X, Huang M, Yang Y, Fayad $\mathrm{ZA}$, Zhang $\mathrm{N}$, et al. Chest $\mathrm{CT}$ findings in coronavirus disease-19 (COVID-19): relationship to duration of infection. Radiology. $2020 \mathrm{Feb}$ 20;295(3):200463.

14 Salehi S, Abedi A, Balakrishnan S, Gholamrezanezhad A. Coronavirus disease 2019 (COVID-19): a systematic review of imaging findings in 919 patients. AJR Am J Roentgenol. 2020 Mar 14:1-7. 